\title{
Rapid Development of Fungicide Resistance by Sclerotinia homoeocarpa on Turfgrass
}

\author{
Young-Ki Jo, Seog Won Chang, Michael Boehm, and Geunhwa Jung
}

First author: Department of Plant Pathology and Microbiology, Texas A\&M University, College Station, TX 77843; second author: Bio Regional Innovation Center, Youngdong University, South Korea; third author: Department of Plant Pathology, The Ohio State University, Columbus, OH 43210; and fourth author: Department of Plant, Soil \& Insect Sciences, University of Massachusetts, Amherst, MA 01003. Accepted for publication 11 August 2008.

\begin{abstract}
Jo, Y.-K., Chang, S. W., Boehm, M., and Jung, G. 2008. Rapid development of fungicide resistance by Sclerotinia homoeocarpa on turfgrass. Phytopathology 98:1297-1304

Dollar spot, caused by Sclerotinia homoeocarpa, is the most prevalent and economically important turfgrass disease in North America. Increasing levels of fungicide resistance, coupled with tightening environmental scrutiny of existing fungicides, has left fewer options for managing dollar spot. More knowledge about $S$. homoeocarpa populations is needed to improve dollar spot management strategies, especially with respect to minimizing the development of fungicide resistance. Population diversity of $S$. homoeocarpa was examined using inter-simple sequence repeat markers and vegetative compatibility assays. Two subgroups were found in $S$. homoeocarpa field populations on both fairway and putting green turfgrass at a research field in Wisconsin. These subgroups were geneti-

cally different, vegetatively incompatible, and had different fungicide sensitivities. The frequency of the two genetic subgroups differed significantly between the fairway and putting green, but was uniform within the fairway or within the green. Population dynamics of $S$. homoeocarpa in response to two systemic fungicides (thiophanate-methyl and propiconazole) were assessed based on in vitro fungicide sensitivity. Dynamics of $S$. homoeocarpa populations depended on the presence of fungicideresistant isolates in the initial populations before fungicide applications and changed rapidly after fungicide applications. Shifting of the population toward propiconazole resistance was gradual, whereas thiophanatemethyl resistance developed rapidly in the population. In conclusion, field populations of $S$. homoeocarpa containing genetically distinct, vegetatively incompatible groups were different on turfgrass that was managed differently, and they were changed rapidly after exposure to fungicides.
\end{abstract}

Dollar spot, caused by Sclerotinia homoeocarpa F. T. Bennett, is the most prevalent and economically important turfgrass disease in North America $(22,23)$. Highly maintained turfgrasses, such as creeping bentgrass (Agrostis stolonifera L.), the most widely used species for golf courses, and Kentucky bluegrass (Poa pratensis L.), the most popular home lawn grass, are susceptible to this disease. To meet the high expectation of aesthetic quality and playability, fungicide input is a requisite for managing dollar spot on manicured turfgrass, even though adequate nitrogen fertility programs and cultural practices designed to reduce leaf wetness may succeed in alleviating some of dollar spot pressure $(4,26)$. Multiple applications of fungicides are usually required throughout the growing season due to the persistent nature of $S$. homoeocarpa.

Numerous fungicides labeled for dollar spot have been released over the past century $(3,23)$. Many of these fungicides, particularly systemic fungicides, successfully controlled dollar spot when they were first released, but $S$. homoeocarpa has developed resistance to several classes of fungicides used for dollar spot control in the United States, including earlier heavy metal-based compounds $(2,18)$, traditional contact fungicides $(21)$, and local or acropetal systemic fungicides such as dicarboximides (6), benzimidazoles $(6,24,25)$, and demethylation inhibitors (DMIs) $(8,11)$. More often than not, $S$. homoeocarpa exhibits crossresistance (i.e., resistance to more than one fungicide within the same chemical group) or multiple resistance (i.e., resistance to different fungicide classes) (23). Jo et al. (13) recently reported

Corresponding author: G. Jung; E-mail address: jung @ psis.umass.edu

doi:10.1094/PHYTO-98-12-1297

(c) 2008 The American Phytopathological Society that resistance to both benzimidazole fungicide thiophanatemethyl and DMI fungicide propiconazole is likely prevalent on golf courses in Ohio. Increasing levels of fungicide resistance, coupled with tightened environmental scrutiny of existing fungicides, has left fewer chemical options for controlling dollar spot.

A population must be the target of a control strategy to successfully manage a plant-pathogenic fungus because the fungal population evolves to survive in a given environment (19). Turfgrass is a perennial system continuously influenced by various management practices, including irrigation, mowing, and the use of fungicides, insecticides, herbicides, and fertilizers. Such agricultural inputs will likely impose strong selection pressures on pathogen populations. Therefore, an understanding of genetic structure and dynamics of pathogen populations is critical for disease management on turfgrass.

Improving chemical and cultural management of dollar spot and delaying the inevitable occurrence of fungicide resistance will require gaining more knowledge about $S$. homoeocarpa populations. The current research first examined genetic diversity and resistance evolution of $S$. homoeocarpa field populations on turfgrass. More specifically, using DNA fingerprinting and vegetative compatibility assays, the population diversity and spatial distribution of $S$. homoeocarpa were examined on turfgrass. Subsequently, population dynamics of $S$. homoeocarpa after exposure to systemic fungicides, which are commonly used for dollar spot, were examined based on in vitro fungicide sensitivity.

\section{MATERIALS AND METHODS}

Establishment and sampling of field plots. Sampling of $S$. homoeocarpa was conducted at the O. J. Noer Turfgrass and Education Facility in Verona, Wisconsin in 2005 and 2006, and 
the Joseph Troll Turf Research Center in South Deerfield, Massachusetts in 2006. Putting greens and fairways in both locations have a mixture of creeping bentgrass and annual bluegrass (Poa annua L.) and have been mowed at a height of 0.3 and $1.3 \mathrm{~cm}$, respectively. The O. J. Noer field was infrequently sprayed with thiophanate-methyl and propiconazole for dollar spot. The Joseph Troll field has never been sprayed with either fungicide.

For the study of genetic diversity and vegetative compatibility of $S$. homoeocarpa field populations, one putting green site $(15 \times$ $15 \mathrm{~m}$; WI-Green) and one fairway site $(15 \times 15 \mathrm{~m}$; WI-Fairway) at the O. J. Noer field were selected in September 2005 for sampling (sampling 1). One hundred dollar spot infection centers were randomly selected from each site and one symptomatic leaf blade from each infection center was used for isolating fungal mycelium using the procedure described in Jo et al. (13).

For examination of spatial distribution of the $S$. homoeocarpa population, the same putting green (WI-Green) and fairway (WIFairway) at the O. J. Noer field were selected for sampling (sampling 2) in June 2006. Samples of leaf blades infected with $S$. homoeocarpa were taken using a hierarchical sampling scheme $(15,19)$ to define the spatial scale that covered the dispersal distance of the fungus. A grid pattern was hierarchically drawn on each turfgrass site at four spatial scales: 15, 3.8, 0.9, and $0.2 \mathrm{~m}$ (Fig. 1). Sample collections were made from dollar spot infection centers nearest to the intersections of each set of grid lines. In total, 100 samples per turfgrass site were collected in the hierarchical grid patterns: 25 samples $3.8 \mathrm{~m}$ apart on $15 \times 15 \mathrm{~m}$; 21 samples $0.9 \mathrm{~m}$ apart on $3.8 \times 3.8 \mathrm{~m} ; 21$ samples $0.2 \mathrm{~m}$ apart on $0.9 \times 0.9$ $\mathrm{m} ; 21$ samples $0.06 \mathrm{~m}$ apart on $0.2 \times 0.2 \mathrm{~m}$; and 12 samples from one dollar spot infection center $(<0.02 \mathrm{~m}$ in diameter).

To determine the effect of repeated fungicide applications on $S$. homoeocarpa populations, field plots were established on the same putting green (WI-Green) and fairway (WI-Fairway) at the O. J. Noer field, and an additional fairway (MA-Fairway) at the Joseph Troll field in 2006. The plots were mowed with separate mowers and clippings were collected. The size of individual plots was $2.7 \times 1.8 \mathrm{~m}$ with $0.9 \mathrm{~m}$ buffer zones between the plots. Plots treated with three systemic fungicides (thiophanate-methyl [3336 WP; Cleary Chemical Corporation, Dayton, NJ], propiconazole [Banner MAXX 1.3ME; Syngenta Professional Products, Greensboro, $\mathrm{NC}]$, and a confidential active ingredient with a different mode of action [CLEX, no registered name; Cleary Chemical Corporation]) and nontreated control plots were arranged in a randomized complete block design with four replicates. One symptomatic leaf blade was collected from each of 10 randomly

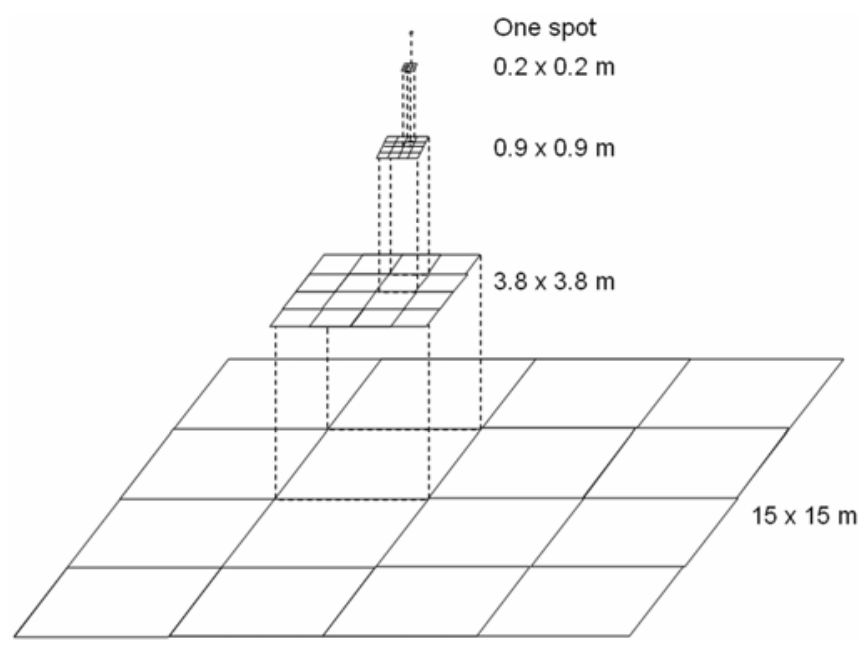

Fig. 1. Hierarchical sampling scheme. Leaf blades with disease symptoms were taken from dollar spot infection centers closest to the intersections of lines of different sized grids: $15 \times 15 \mathrm{~m}, 3.8 \times 3.8 \mathrm{~m}, 0.9 \times 0.9 \mathrm{~m}, 0.2 \times 0.2 \mathrm{~m}$, and one dollar spot patch $(0.02 \mathrm{~m}$ in diameter). selected dollar spot infection centers per plot before each fungicide application (sampling 3).

The spray volume was equivalent to $81.5 \mathrm{ml} / \mathrm{m}^{2}$ and was applied at $275.8 \mathrm{kPa}$ with a $\mathrm{CO}_{2}$-pressured boom sprayer equipped with two XR Teejet 8005 VS nozzles. Fungicide applications began in June 2006 for the plots at the O. J. Noer field and in August for the plots at the Joseph Troll field and were repeated with an approximately 21-day interval until September 2006. In cases where there were no disease symptoms on fungicide-treated plots, the fungicide application was postponed until dollar spot infection centers appeared or until the next application date.

In vitro sensitivity to fungicides. $S$. homoeocarpa isolates were recovered from individual symptomatic leaf blades and were used for assaying in vitro sensitivity to thiophanate-methyl, propiconazole, and CLEX using the procedure previously described by Jo et al. (13). Autoclaved potato dextrose agar (PDA; Becton, Dickson and Company, Sparks, MD) media were amended with the following single discriminatory concentrations of these fungicides previously determined by Jo et al. (13) and our preliminary test: $1,000 \mu \mathrm{g}$ of active ingredient (a.i.)/ml of thiophanate-methyl; $0.1 \mu \mathrm{g}$ a.i./ml of propiconazole; and 1,000 $\mu \mathrm{g}$ a.i./ml of CLEX. Thiophanate-methyl- or CLEX-amended PDA was poured into sterile 6-cm-diameter petri dishes, and propiconazole-amended PDA was poured into sterile $10-\mathrm{cm}$-diameter petri dishes.

To measure the inhibition of mycelial growth by fungicides, agar plugs ( $5 \mathrm{~mm}$ in diameter) were taken from the edge of actively growing 3-day-old colonies of each isolate and placed onto fungicide-amended PDA and nonamended PDA as the control. Then, the culture plates were incubated at $25^{\circ} \mathrm{C}$ for $30 \mathrm{~h}$. We examined the growth of mycelium in a qualitative term of whether mycelium on thiophanate-methyl or CLEX-amended PDA grew or not. For propiconazole, two perpendicular diameters of the colonies were measured and averaged. The percentage of relative mycelial growth (RMG) on propiconazole-amended PDA compared with normal growth on nonamended PDA was determined and used as an indicator of sensitivity of the pathogen to propiconazole.

In vitro fungicide sensitivities of each isolate were measured at the single concentration of each of three fungicides. This test was conducted twice. In vitro sensitivity data for each fungicide was subjected to a separate analysis of variance.

Genetic diversity. The genetic diversity of $S$. homoeocarpa populations on the fairway and putting green at the O. J. Noer field in 2005 was analyzed using inter-simple sequence repeat (ISSR) markers $(10,27)$. A total of 52 and 69 isolates were randomly selected as a representative of the populations on WIGreen and WI-Fairway (sampling 1). Each of these isolates was grown on PDA at $25^{\circ} \mathrm{C}$ for 7 days. Mycelium was harvested and placed in $600 \mu \mathrm{l}$ of lysis buffer $(100 \mathrm{mM}$ Tris, $\mathrm{pH} 8.0 ; 50 \mathrm{mM}$ EDTA; $500 \mathrm{mM} \mathrm{NaCl}$; and $10 \mathrm{mM} \beta$-mercaptoethanol) in 2-ml plastic tubes containing small metal beads $(4.5 \mathrm{~mm}$ in diameter). The tray of tubes was placed in a pulverizing machine (Biospec Products Inc., Bartlesville, OK) and rapid shaking of beads broke cells. DNA was extracted using the Dellaporta extraction method (5).

Four ISSR primers, $\mathrm{T}(\mathrm{CT})_{7} \mathrm{CRT}, \mathrm{HBH}(\mathrm{AG})_{7}, \mathrm{VDV}(\mathrm{CT})_{7}$, and $\operatorname{DVD}(\mathrm{TC})_{7}$, which were found to be most informative in a preliminary screening, were used. The primers were synthesized by Integrated DNA Technologies, Inc. (Coralville, IA). Polymerase chain reaction (PCR) was performed with a Thermal Controller (MJ Research Inc., Watertown, MA) in a 10- $\mu$ l volume containing $4 \mathrm{ng}$ of fungal genomic DNA, $1.0 \mu \mathrm{M}$ concentration of each ISSR primer, $0.2 \mathrm{mM}$ of each deoxynucleoside triphosphate, $2.0 \mathrm{mM}$ $\mathrm{MgCl}_{2}, 1 \times \mathrm{Taq}$ polymerase buffer, and $5 \mathrm{U}$ of $\mathrm{Taq}$ polymerase. PCR was run under the following conditions: an initial preheating for $1 \mathrm{~min}$ at $95^{\circ} \mathrm{C}$, followed by 40 cycles of denaturation at $91^{\circ} \mathrm{C}$ for $15 \mathrm{~s}$, annealing at $42^{\circ} \mathrm{C}$ for $15 \mathrm{~s}$, and extension at $72^{\circ} \mathrm{C}$ for $1 \mathrm{~min}$, and $10 \mathrm{~s}$ with a final extension at $72^{\circ} \mathrm{C}$ for $10 \mathrm{~min}$. PCR 
was performed twice for each isolate. Amplicons were separated on $1.5 \%$ agarose gels in Tris-borate-EDTA buffer and photographed after being stained with ethidium bromide.

For estimating genetic similarity, the presence or absence of each amplicon was visually scored for each isolate, since ISSR are considered dominant markers. Genetic similarity $(S)$ was calculated by the formula $S=2 N_{x y} /\left(N_{x}+N_{y}\right)$, where $N_{x y}$ is the number of bands in common and $N_{x}$ and $N_{y}$ are the numbers of bands found in isolates $x$ and $y$, respectively (17). This genetic distance data was then used to create a multidimensional scaling (MDS) plot using PROC MDS in SAS package (SAS Institute Inc., Cary, NC). In addition, analysis of molecular variance (AMOVA) was conducted to determine the genetic diversity between different genetic subgroups. Gene diversity $(H)$ within each genetic subgroup was estimated by the following formula:

$$
H=\frac{n}{(n-1)}\left(1-\sum_{i=1}^{k} P_{i}^{2}\right)
$$

where $n$ is the number of gene copies in the sample, $k$ is the number of haplotypes, and $P_{i}$ is the sample frequency of the $i$ th haplotype (20). AMOVA and gene diversity $(H)$ analyses were implemented using Arlequin version 3.1 software (Computational and Molecular Population Genetics Lab, Bern, Switzerland).

Vegetative compatibility. Fifteen representative $S$. homoeocarpa isolates having a different range of in vitro fungicide sensitivities, which were collected from the fairway and putting green at the O. J. Noer field in 2005 (sampling 1), were selected for the vegetative compatibility assay. Nitrate nonutilizing (nit) mutants were recovered from each isolate according to the procedure described by Jo et al. (12).

Vegetative compatibility was determined based on complementary heterokaryon formation in pairings between two nit mutants derived from the $15 \mathrm{~S}$. homoeocarpa isolates. Agar plugs $(5 \mathrm{~mm}$ in diameter) were cut from the edge of each nit mutant colony growing on Czapek solution agar (CDA; Becton, Dickson, and Company, Sparks, MD) and paired $4 \mathrm{~cm}$ apart in a 9-cm-diameter plate of CDA with all possible combination between available nit mutants. The plates were incubated at $23^{\circ} \mathrm{C}$ for 14 days. A complementary reaction was evident by the development of dense aerial growth of prototrophic mycelial where paired mutants contacted each other. This experiment was conducted twice.

\section{RESULTS}

Subgroups. Nineteen polymorphic bands generated from four ISSR primers were used to examine genetic diversity of $121 \mathrm{~S}$. homoeocarpa isolates (52 isolates from WI-Green and 69 from WI-Fairway) from populations (sampling 1) at the O. J. Noer field in 2005. These isolates were separated into two genetically distinct groups, designated as subgroups $\alpha$ and $\beta$ (Fig. 2). These two subgroups were present on both WI-Green and WI-Fairway but existed in different frequencies, subgroups $\alpha: \beta=14.5: 85.5 \%$ on WI-Green and 56.5:43.5\% on WI-Fairway. AMOVA between the two subgroups indicated significant difference in genetic variation $(F=0.78 ; P<0.0001)$. The mean genetic variation $(H)$ was greater in subgroup $\alpha(H=0.27 \pm 0.14)$ than subgroup $\beta(H=$ $0.05 \pm 0.11$ ).

The two genetic subgroups showed different sensitivities to thiophanate-methyl and propiconazole (Fig. 2). Isolates in subgroup $\alpha$ from both WI-Green and WI-Fairway were completely resistant to thiophanate-methyl and highly resistant to propiconazole $(\mathrm{RMG}=45.7 \pm 11.5 \%)$. In contrast, most isolates $(83 \%)$ in subgroup $\beta$ were sensitive to both thiophanate-methyl and propiconazole $(\mathrm{RMG}=11.9 \pm 7.0 \%)$. Some isolates $(17 \%)$ in subgroup $\beta$ collected from WI-Fairway were resistant to thiophanate-methyl but still sensitive to propiconazole $(\mathrm{RMG}=10.8 \pm 8.5 \%)$. All isolates in both subgroups were sensitive to CLEX.

Three types of nit mutants (nit1, nit3, and NitM) were obtained from $15 S$. homoeocarpa isolates. With the confirmation of complementary heterokaryon formation between nit mutants, these 15 isolates were separated into two vegetative compatibility groups, which corresponded to subgroups $\alpha$ and $\beta$ (Table 1). Complementary heterokaryons formed only between different mutant phenotypes derived from the same subgroup. Particularly strong complementary reactions were observed between nitl and NitM as previously described (12).
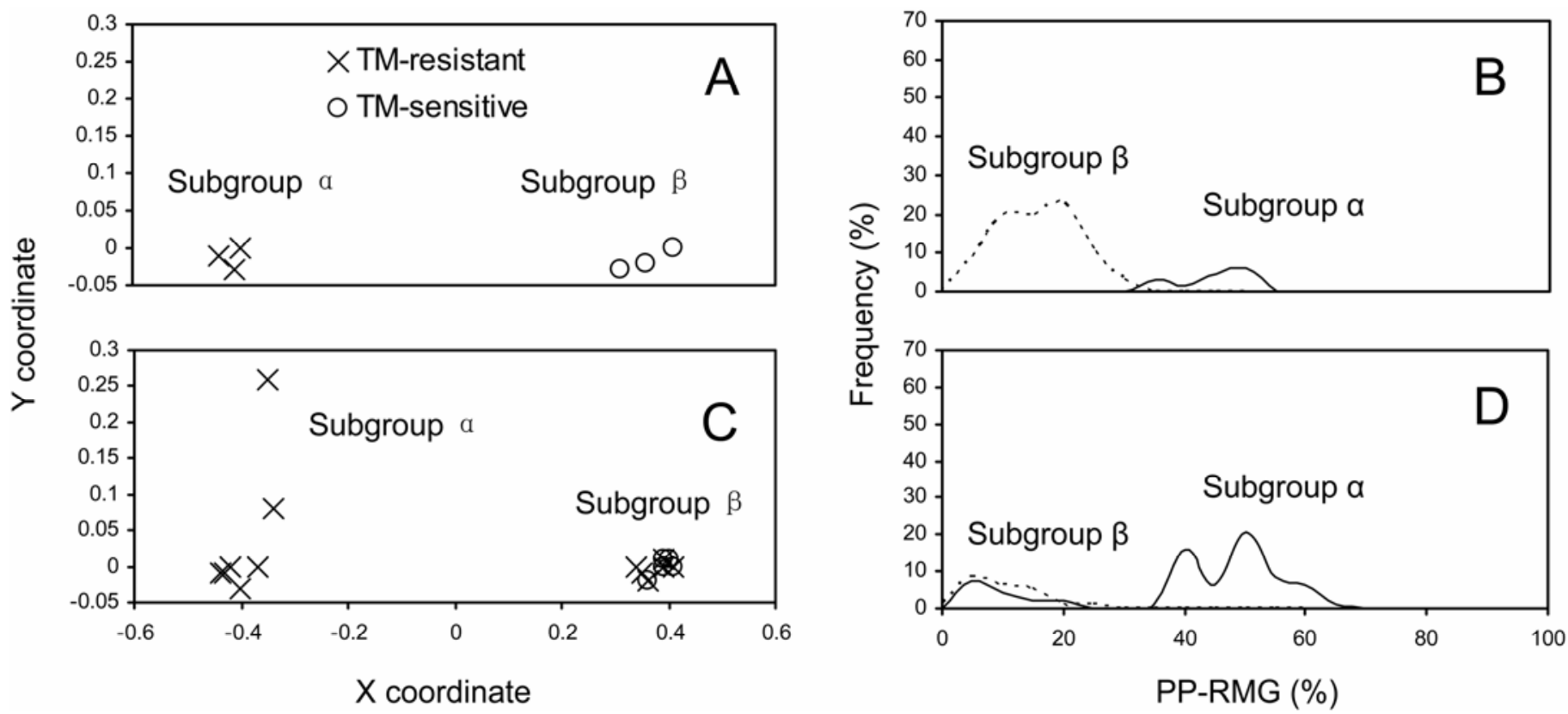

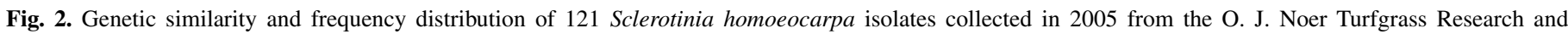

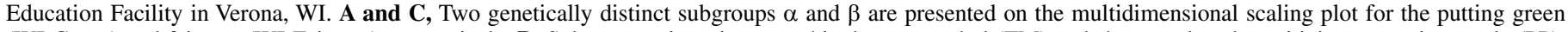

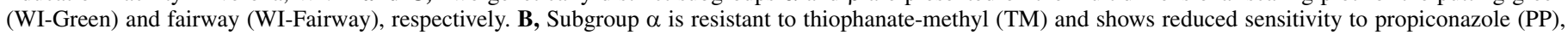

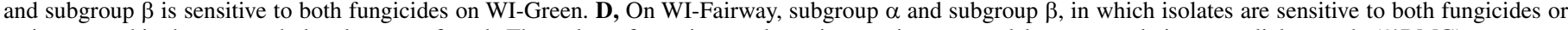

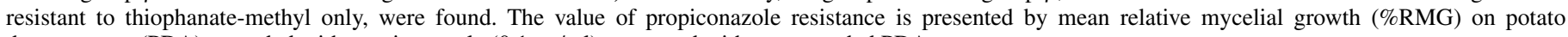
dextrose agar (PDA) amended with propiconazole $(0.1 \mu \mathrm{g} / \mathrm{ml})$ compared with nonamended PDA. 
Spatial distribution of $S$. homoeocarpa. In vitro fungicide sensitivity assay was conducted using $S$. homoeocarpa isolates hierarchically sampled from WI-Fairway and WI-Green sites in 2006 (sampling 2). Spatial distribution of S. homoeocarpa was uniform within the same turfgrass site. Consequently, mean sensitivity values to thiophanate-methyl and propiconazole were distributed evenly within each site (Table 2). For example on WIGreen, the frequency of thiophanate-methyl-resistant isolates consistently remained at 50 to $69 \%$ and the propiconazole sensitivity (32 to $38 \%$ of RMG) did not significantly change in the sample sets hierarchically taken at $15 \times 15 \mathrm{~m}, 3.8 \times 3.8 \mathrm{~m}$, and $0.9 \times$ $0.9 \mathrm{~m}$ grid sizes $(P>0.05)$ (Table 2$)$.

However, frequency of sensitive and resistant isolates was significantly different among the populations on WI-Fairway and WI-Green. Thiophanate-methyl-resistant isolates accounted for $86 \%$ of the population on WI-Fairway and $61 \%$ on WI-Green. Mean RMG indicative of propiconazole resistance was significantly higher for the populations from WI-Green $(\mathrm{RMG}=$ $37.8 \pm 13.9)$ than that from WI-Fairway $(\mathrm{RMG}=20.4 \pm 15.7)$ $(P<0.05)$.

Population dynamics in response to repeated applications of fungicides. Applications of propiconazole and CLEX significantly reduced dollar spot severity on WI-Green (Fig. 3A), WIFairway (Fig. 3B), and MA-Fairway (Fig. 3C). The thiophanatemethyl treatment significantly reduced dollar spot severity on MA-Fairway but not on WI-Green or WI-Fairway. In vitro fungicide sensitivities of the populations on WI-Green, WI-Fairway, and MA-Fairway in 2006 (sampling 3) changed differently, depending on types of treated fungicides and turfgrass sites.
On WI-Green, four repeated applications of thiophanate-methyl or propiconazole and three repeated applications of CLEX significantly increased the frequency of isolates resistant to thiophanatemethyl (Fig. 3D) and/or isolates resistant to propiconazole (Fig. $3 \mathrm{G})$ in the population $(P<0.05)$ compared with the nontreated control plot. On WI-Fairway, three repeated applications of thiophanate-methyl or propiconazole and two repeated applications of CLEX increased the frequency of thiophanate-methyl-resistant isolates to almost $100 \%$ in the population (Fig. 3E). Propiconazole applications significantly shifted the population toward propiconazole resistance at the end of the season compared with the nontreated control plot $(P<0.05)$. However, applications of thiophanate-methyl or CLEX did not influence propiconazole sensitivity of the population (Fig. 3H). On MA-Fairway, two repeated applications of thiophanate-methyl dramatically increased the frequency of thiophanate-methyl-resistant isolates from $0 \%$ at the initial sample set to $100 \%$ at the last sample set (Fig. 3F). However, applications of any of the three fungicides did not change propiconazole sensitivity of the population on MAFairway (Fig. 3I).

\section{DISCUSSION}

Genetic diversity and fungicide resistance evolution of $S$. homoeocarpa field populations were examined for the first time based on in vitro fungicide sensitivity, DNA fingerprinting, and vegetative compatibility. The $S$. homoeocarpa population on a turfgrass research field in Wisconsin consisted of two subgroups, which were genetically different, vegetatively incompatible, and

TABLE 1. Vegetative compatibility between Sclerotinia homoeocarpa isolates collected from the fairway and putting green at the O. J. Noer Turfgrass Research and Education Facility in Verona, WI, in June 2006 using nitrate nonutilizing (nit) mutants

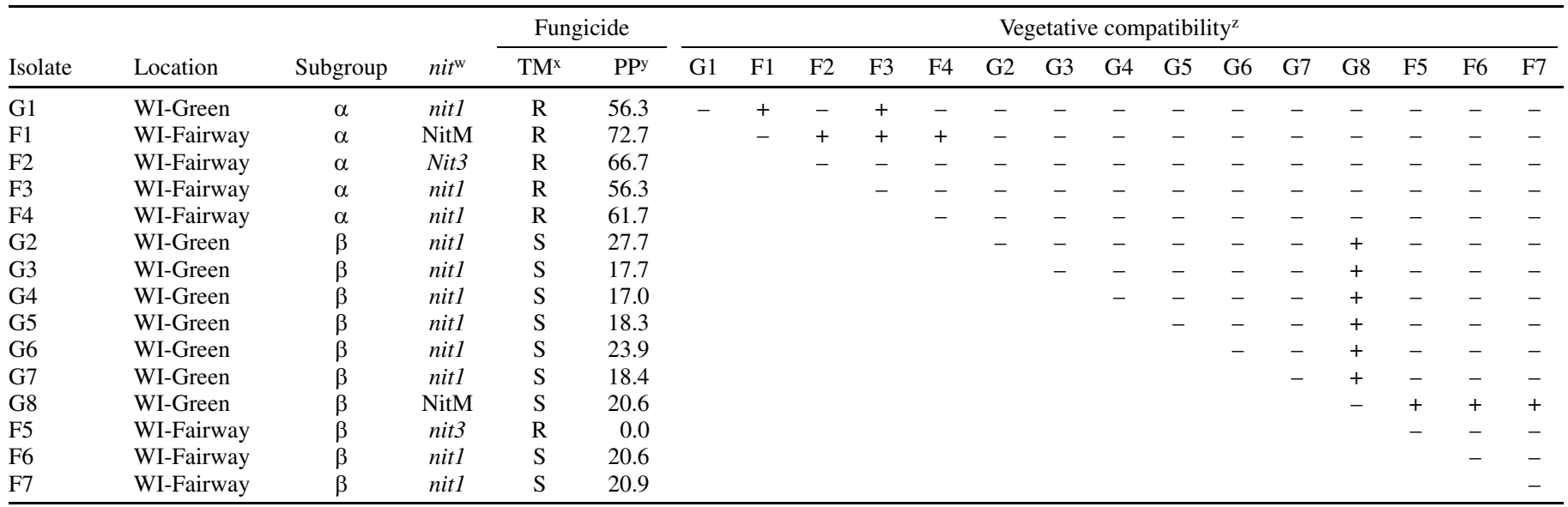

${ }^{\mathrm{w}}$ Nit mutant phenotype based on the growth on Czapek solution agar media with different nitrogen sources (12).

${ }^{\mathrm{x}}$ Resistant (R) or susceptible (S) to thiophanate-methyl (TM).

y Mean percent relative mycelial growth (RMG) on the growth media amended with propiconazole (PP; $0.1 \mu \mathrm{g} / \mathrm{ml})$ compared with unamended media.

$\mathrm{z}^{\mathrm{z}}=$ complementation; and $-=$ no complementation.

TABLE 2. In vitro fungicide sensitivities and subgroups of Sclerotinia homoeocarpa isolates collected from the fairway and putting green at the O. J. Noer Turfgrass Research and Education Facility in Verona, WI, in June 2006 using the hierarchical sampling scheme

\begin{tabular}{|c|c|c|c|c|c|c|c|c|}
\hline \multirow[b]{2}{*}{ Grid size $(m \times m)$} & \multirow{2}{*}{$\begin{array}{c}\text { Spatial } \\
\text { distance }(\mathrm{m})\end{array}$} & \multirow{2}{*}{$\begin{array}{l}\text { Sample } \\
\text { number }\end{array}$} & \multicolumn{3}{|c|}{ WI-Fairway } & \multicolumn{3}{|c|}{ WI-Green } \\
\hline & & & $\mathrm{TM}^{\mathrm{x}}$ & $\mathrm{PP}^{\mathrm{y}}$ & Subgroup $\alpha^{z}$ & $\mathrm{TM}$ & PP & Subgroup $\alpha$ \\
\hline $15.0 \times 15.0$ & 3.8 & 25 & 82.6 & $27.2 \pm 16.8 \mathrm{a}$ & 54.5 & 50.0 & $31.7 \pm 14.2 \mathrm{a}$ & 50.0 \\
\hline $3.8 \times 3.8$ & 0.9 & 21 & 80.0 & $18.9 \pm 15.1 \mathrm{a}$ & 30.0 & 68.8 & $38.0 \pm 13.6 \mathrm{a}$ & 68.8 \\
\hline $0.2 \times 0.2$ & 0.06 & 21 & 90.5 & $22.2 \pm 16.9 \mathrm{a}$ & 38.1 & 100.0 & $49.4 \pm 5.2 b$ & 100.0 \\
\hline $\begin{array}{l}\text { One spot } \\
(0.02 \mathrm{~m} \text { in diam }\end{array}$ & & 12 & 100.0 & $8.5 \pm 3.1 \mathrm{~b}$ & 0.0 & 100.0 & $46.0 \pm 3.2 \mathrm{~b}$ & 100.0 \\
\hline
\end{tabular}

${ }^{\mathrm{x}}$ Percentage of resistant isolates to thiophanate-methyl (TM).

${ }^{y}$ Mean and standard deviation of percent relative mycelial growth (RMG) on growth media amended with propiconazole (PP; $\left.0.1 \mu \mathrm{g} / \mathrm{ml}\right)$ compared with mycelial growth on unamended media. Same letters within a column indicate no significant difference using Fisher's protected least significance difference at $P=0.05$.

z Percentage of isolates grouped into subgroup $\alpha$, which was determined by reduced sensitivity to propiconazole (RMG $>30 \%$ ). 
had different fungicide sensitivities. These subgroups were significantly different in frequency between the putting green and fairway which were managed differently but were uniformly distributed within the same turfgrass site. The $S$. homoeocarpa populations changed rapidly toward fungicide resistance after repeated applications of systemic fungicides. The current comprehensive investigation about population dynamics of $S$. homoeocarpa provides the vital information for future improvement of chemical and cultural management strategies for dollar spot with a minimal risk of resistance development.

Two genetic subgroups based on ISSR markers were detected in the S. homoeocarpa population at the O. J. Noer field. Interestingly, each subgroup was linked with different phenotypes such as fungicide sensitivity and vegetative compatibility. Particularly, in vitro sensitivities to propiconazole were very different between these two subgroups (Table 2). Isolates in subgroup $\alpha$ were more resistant to propiconazole ( $\mathrm{RMG}>30 \%$ ) than those in subgroup $\beta$ $(\mathrm{RMG}<30 \%)$. Furthermore, these two subgroups were vegetatively incompatible with each other, indicating two clonal lineages originating from different progenitors. Isolates within the same subgroup produced stable hyphal anastomoses, ensuring potential genetic exchange and diversity buildup. In contrast, isolates between different subgroups acted to restrict the formation of heterokaryons so that they might compete with each other.
With no previous information about the population structure, the hierarchical sampling scheme employed in this study revealed new information on a spatial distribution of $S$. homoeocarpa field populations on turfgrass (Table 2). S. homoeocarpa scattered in small, noncontiguous spots that were spatially disconnected from their points of origin and were uniformly mixed on turfgrass treated with the same management practices including mowing, irrigation, and chemical input. Similar patterns of spatial distribution have been reported from other plant-pathogenic fungi in agrosystems including $S$. sclerotiorum in canola $(1,15)$ and $F u$ sarium oxysporum in cultivated soils (9). Although S. homoeocarpa does not disseminate via either sexual or asexual spores, cultural practices employed on turfgrass, most likely frequent mowing by the same mower, may play a significant role in a uniform dispersal of $S$. homoeocarpa on turfgrass. Therefore, sampling of $S$. homoeocarpa at relatively small spatial scales (the minimum plot size of $0.9 \times 0.9 \mathrm{~m}$ ) seems to be enough to represent the individual local population, which is valuable information for future sampling of $S$. homoeocarpa in various fields.

The $S$. homoeocarpa population structure was significantly different on turfgrass sites managed differently, suggesting that unique management practices employed on different turfgrass sites greatly affect fungal populations. Mowing (frequency and height) and chemical input (fertilizer, herbicide, insecticide, and
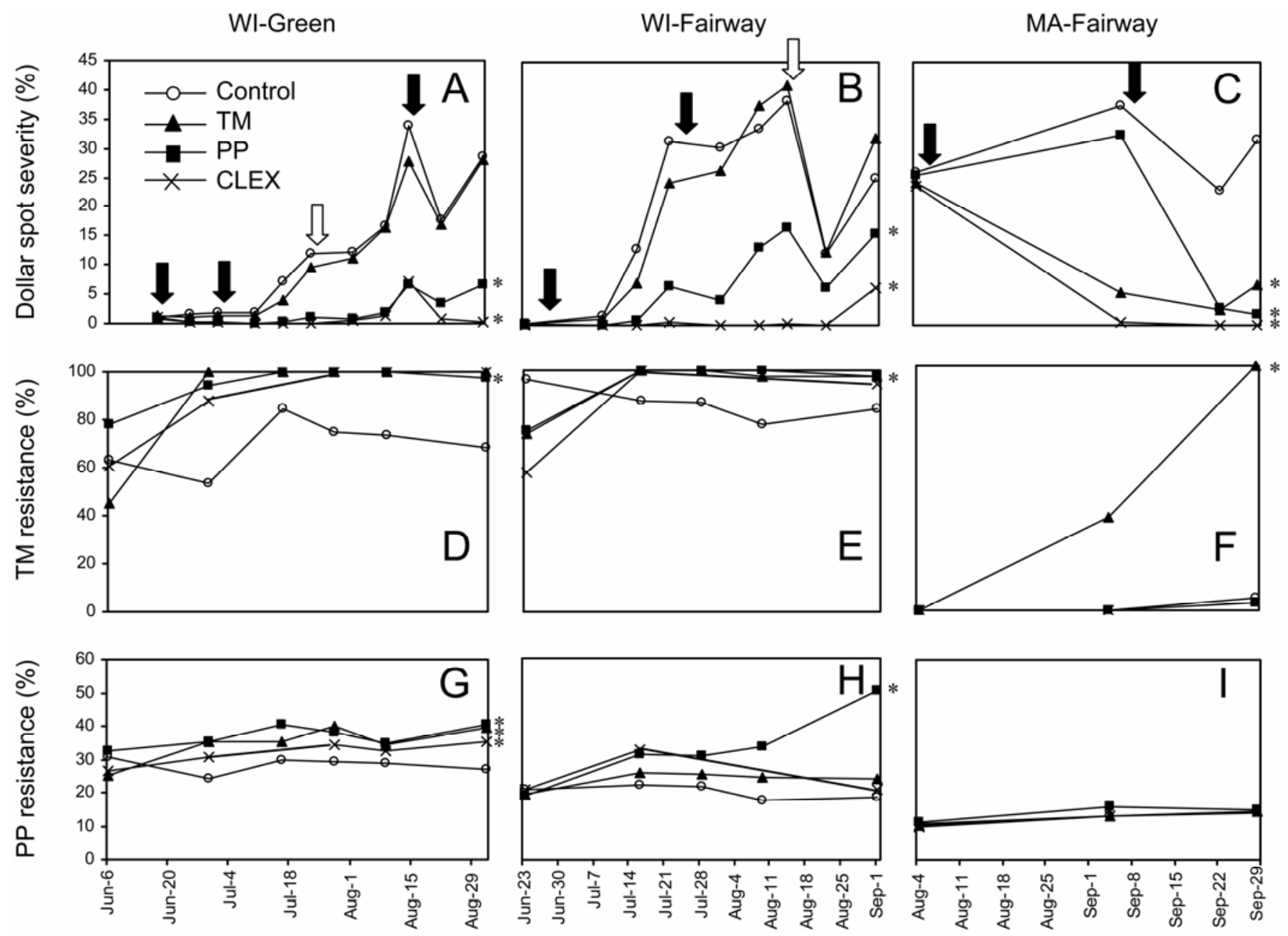

Fig. 3. Time-course changes of dollar spot severity and fungicide sensitivity on the plots treated with thiophanate-methyl (TM), propiconazole (PP) or CLEX, and nontreated control plots. A, B, and C, The plots were established in 2006 on the green (WI-Green) and fairway (WI-Fairway) in Verona, WI and the fairway (MAFairway) in South Deerfield, MA, respectively. Black arrows indicate application dates of three fungicides and white arrows indicate application dates of thiophanate-methyl and propiconazole only. $\mathbf{D}, \mathbf{E}$, and $\mathbf{F}$, Thiophanate-methyl resistance is presented as the percentage of resistant isolates in the total isolates tested at these three locations. The thiophanate-methyl-resistant isolates grow on potato dextrose agar (PDA) amended with thiophanate-methyl (1,000 $\mu \mathrm{g} / \mathrm{ml})$. G, H, and I, Propiconazole sensitivity is presented by mean relative mycelial growth (\%RMG) of Sclerotinia homoeocarpa isolates collected from each plot at the three locations. Asterisks indicate that the value on the plot is significantly different compared with the nontreated control plots at the end of the season using Fisher's protected least significance difference at $P=0.05$. 
Time 0
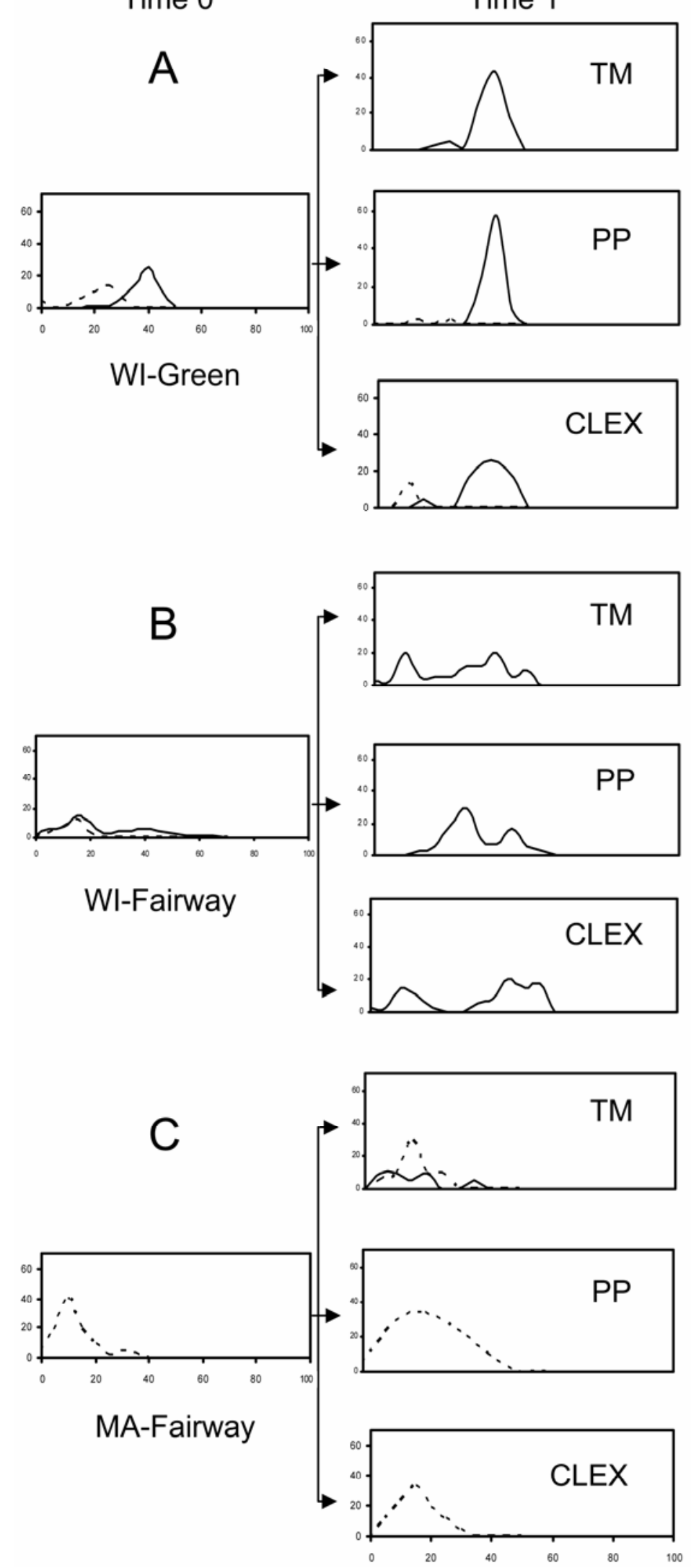

Time 2
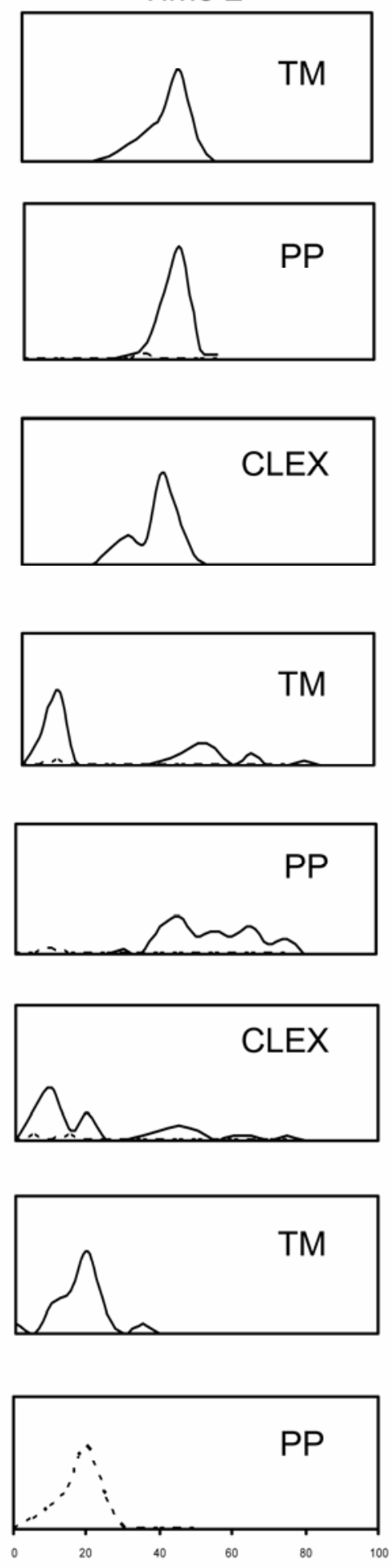

TM-resistant

\section{TM-sensitive}

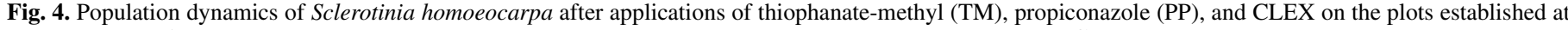

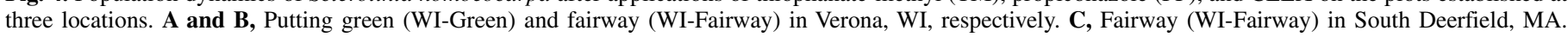

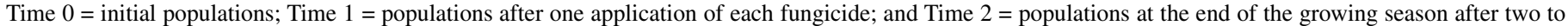

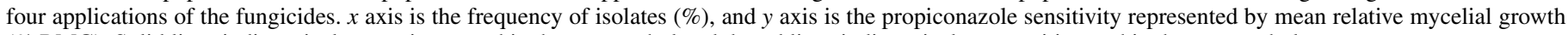
(\% RMG). Solid lines indicate isolates resistant to thiophanate-methyl and dotted lines indicate isolates sensitive to thiophanate-methyl. 
fungicide) are more intensive on the putting green than the fairway and rough on golf courses. Disease progression of $S$. homoeocarpa is often different between the fairway and adjacent putting green. The current study showed that dollar spot progressed relatively later and severer on the fairway (Fig. 3A, WIFairway) than the putting green (Fig. 3B, WI-Green) at the O. J. Noer field in 2006. In another survey study of $S$. homoeocarpa fungicide resistance on six golf courses in Wisconsin and one golf course in Massachusetts, in vitro sensitivities for thiophanatemethyl and propiconazole were significantly different among the fairway, putting green, and rough within the same golf course (14). However, direct evidence of how specific factors of management practices can affect fungicide sensitivity of $S$. homoeocarpa populations has not been examined experimentally and remains in need of further research.

Based on dynamics of different individual $S$. homoeocarpa populations in response to systemic fungicides, evolution of populations toward multiple resistance to benzimidazole (thiophanate-methyl) and DMI (propiconazole) fungicides in turfgrass agrosystems can be envisioned. The initial population on MAFairway where no fungicide has been used before may represent a wild-type population. All $S$. homoeocarpa isolates sampled at the beginning of the field study were sensitive to both thiophanatemethyl and propiconazole (Time 0, Fig. 4C). After two applications of thiophanate-methyl, isolates resistant to thiophanatemethyl were rapidly selected up to $100 \%$ in frequency and became dominant in the population at the end of the growing season. In contrast, propiconazole applications did not affect propiconazole sensitivity of the population. Consequently, isolates resistant or sensitive to thiophanate-methyl coexisted in the population but both types were still highly sensitive to propiconazole (Fig. 4C, PP). This observation indicates that benzimidazole resistance can be selected more quickly and earlier in a wild-type S. homoeocarpa field population than DMI resistance.

The $S$. homoeocarpa populations consisting of subgroups $\alpha$ and $\beta$ in the O. J. Noer field may represent populations which had already developed fungicide resistance after previous exposure to fungicides. In the aspect of coexistence of resistant and sensitive isolates to thiophanate-methyl and still remaining of high sensitivity to propiconazole, subgroup $\beta$ found on WI-Fairway (Fig. 2D) is similar to the new population on MA-Fairway at the end of the growing season in 2006. Subgroup $\alpha$ found on WI-Green and WI-Fairway would be further evolved toward fungicide resistance because of highly reduced sensitivity to both thiophanate-methyl and propiconazole. It is hypothesized that thiophanate-methyl resistance was first selected and subsequently propiconazole resistance developed in subgroup $\alpha$. This hypothesis is also in agreement with Fisher's fundamental theorem saying that the evolutionary potential of a population is proportional to the amount of genetic diversity in a population (7). Subgroup $\alpha$ contains greater genetic variation than those in subgroup $\beta$, indicating a greater chance for the accumulation of mutations in subgroup $\alpha$ conferring fungicide resistance. Our following-up genetic study on the population of MA-Fairway may elucidate this hypothesis.

Questions of how mutation accumulates for DMI resistance in the population or how long it takes are beyond the scope of this study. However, the current investigation demonstrated that development of DMI resistance is more gradual and takes longer than benzimidazole resistance. This result makes sense since resistance to DMIs is known to be quantitatively controlled and requires multiple mutations, while single point mutations at the $\beta$ tubulin gene cause complete resistance to benzimidazole (16). On MA-Fairway during one growing season, two applications of propiconazole did not change the propiconazole sensitivity of the population (Fig. 4C, PP), while two repeated applications of thiophanate-methyl rapidly selected isolates that are completely resistant to thiophanate-methyl (Fig. 4C, TM). Isolates collected from WI-Fairway (Fig. 4B, PP) had a wide range of propi- conazole resistance, indicating the resistance is controlled by multiple genes. Propiconazole resistance of the population gradually increased as highly resistant isolates became selected by repeated applications of propiconazole.

Population dynamics and fungicide efficacy in the field seem to be directly dependent on types of fungicides applied and the initial $S$. homoeocarpa population before fungicide application. This provides inference for the successful use of fungicides for dollar spot control. Since rapid development of resistance to thiophanate-methyl is possible despite few resistant isolates present in the initial population (Fig. 4C, TM), repeated applications of thiophanate-methyl for dollar spot control should be avoided at any case. In the case of propiconazole, complete failure of the fungicide may not occur because the fungicide still can reduce dollar spot caused by resistant isolates. However, efficacy of propiconazole for dollar spot control may significantly diminish as highly resistant isolates are selected in the population, shown in propiconazole-treated plots on WI-Fairway (Fig. 4B, PP). Therefore, repeated applications of propiconazole also should be avoided if isolates with high resistance to propiconazole are already found in the initial population.

As an alternative to the repeated application with a single chemical, a rotation of thiophanate-methyl and propiconazole may be more effective unless isolates with multiple resistance to both fungicides, like subgroup $\alpha$, exist in the population. CLEX, a new fungicide chemistry, worked well for reducing dollar spot caused by resistant isolates to thiophanate-methyl and propiconazole as shown in CLEX-treated plots on WI-Green (Fig. 3A) and WI-Fairway (Fig. 3B). However, it may still select isolates resistant to thiophanate-methyl and/or propiconazole in the population as shown on WI-Green (Fig. 4A, CLEX) and WI-Fairway (Fig. 4B, CLEX) for unknown reasons.

The current study shows that S. homoeocarpa populations on turfgrass shift fast toward resistance to systemic fungicides, which may explain prevalent distribution of fungicide resistant $S$. homoeocarpa on golf courses in Ohio (13) and Wisconsin (14). This result also indicates that the $S$. homoeocarpa field populations continuously change and adapt to varying environmental conditions. Further long-term research about the effects of fungicides and cultural practices on the S. homoeocarpa population will increase knowledge about the population dynamics of phytopathogenic fungi on turfgrass.

\section{ACKNOWLEDGMENTS}

We thank P. Koch, J. Rees, R. Escalante, N. Beaulieu, and D. Golding for maintaining field plots, collecting samples, and preparing assays; $\mathrm{M}$. Casler for advice on statistics; and K. Hutchins and L. Adler for editorial review of the manuscript. This project was supported by Cleary and Syngenta and partially by the start-up funds from Department of Plant, Soil, and Insect Sciences, University of Massachusetts-Amherst.

\section{LITERATURE CITED}

1. Anderson, J. B., and Kohn, L. M. 1995. Clonality in soilborne, plantpathogenic fungi. Annu. Rev. Phytopathol. 33:369-391.

2. Cole, H., Taylor, B., and Duich, J. 1968. Evidence of differing tolerances to fungicides among isolates of Sclerotinia homoeocarpa. Phytopathology 58:683-686.

3. Couch, H. B. 1995. Diseases of Turfgrasses. 3rd ed. Krieger Publishing Co., Malabar, FL.

4. Davis, J. G., and Dernoeden, P. H. 2002. Dollar spot severity, tissue nitrogen, and soil microbial activity in bentgrass as influenced by nitrogen source. Crop Sci. 42:480.

5. Dellaporta, S. L., Wood, J., and Hicks, J. B. 1983. A plant DNA minipreparation: Version II. Plant Mol. Biol. Rep. 1:19-21.

6. Detweiler, A. R., Vargas, J. M., Jr., and Danneberger, T. K. 1983. Resistance of Sclerotinia homoeocarpa to iprodione and benomyl. Plant Dis. 67:627-630.

7. Fisher, R. A. 1930. The Genetical Theory of Natural Selection. 1st ed. Clarendon Press, Oxford. 
8. Golembiewski, R. C., Vargas, J. M., Jr., Jones, A. L., and Detweiler, A. R. 1995. Detection of demethylation inhibitor (DMI) resistance in Sclerotinia homoeocarpa populations. Plant Dis. 79:491-493.

9. Gordon, T. R., Okamoto, D., and Milgroom, M. G. 1992. The structure and interrelationship of fungal populations in native and cultivated soils. Mol. Ecol. 1:241-249.

10. Gupta, M., Chyi, Y.-S., Romero-Severson, J., and Owen, J. 1994. Amplification of DNA markers from evolutionarily diverse genomes using single primers of simple-sequence repeats. Theor. Appl. Genet. 89:998-1006.

11. Hsiang, T., Yang, L., and Barton, W. 1998. Relative virulence of isolates of Sclerotinia homoeocarpa with varying sensitivity to propiconazole. Eur. J. Plant Pathol. 104:163-169.

12. Jo, Y.-K., Chang, S. W., Rees, J., and Jung, G. 2008. Reassessment of vegetative compatibility of Sclerotinia homoeocarpa using nitratenonutilizing mutants. Phytopathology 98:108-114.

13. Jo, Y.-K., Niver, A. L., Rimelspach, J. W., and Boehm, M. J. 2006. Fungicide sensitivity of Sclerotinia homoeocarpa from golf courses in Ohio. Plant Dis. 90:807-813.

14. Koch, P. L., Grau, C. R., Jo, Y., and Jung, G. Thiophanate-methyl and propiconazole sensitivity of Sclerotinia homoeocarpa isolates collected from golf course putting greens, fairways, and roughs in Wisconsin and Massachusetts. Plant Dis. (In Press.)

15. Kohli, Y., Brunner, L. J., Yoell, H., Milgroom, M. G., Anderson, J. B., Morrall, R. A. A., and Kohn, L. M. 1995. Clonal dispersal and spatial mixing in populations of the plant pathogenic fungus, Sclerotinia sclerotiorum. Mol. Ecol. 4:69-77.

16. Köller, W., and Scheinpflug, H. 1987. Fungal resistance to sterol biosynthesis inhibitors: A new challenge. Plant Dis. 71:1066-1074.

17. Lynch, M. 1990. The similarity index and DNA fingerprinting. Mol. Biol.
Evol. 7:478-484

18. Massie, L. B., Cole, H., and Duich, J. 1968. Pathogen variation in relation to disease severity and control of sclerotinia dollarspot of turfgrass by fungicides. Phytopathology 58:1616-1619.

19. McDonald, B. 1997. The population genetics of fungi: Tools and techniques. Phytopathology 87:448-453.

20. Nei, M. 1987. Molecular Evolutionary Genetics. Columbia University Press, New York.

21. Nicholson, J. F., Meyer, W. A., Sinclair, J. B., and Butler, J. D. 1971. Turf isolates of Sclerotinia homoeocarpa tolerant to Dyrene. J. Phytopathol. 72:169-172.

22. Smiley, R. W., Dernoeden, P. H., and Clarke, B. B. 2005. Compendium of Turfgrass Diseases. 3rd ed. American Phytopathological Society, St. Paul, MN.

23. Vargas, J. M., Jr. 1994. Management of Turfgrass Diseases. 2nd ed. Lewis Publishers, Boca Raton, FL.

24. Warren, C. G., Sanders, P., and Cole, H. 1974. Sclerotinia homoeocarpa tolerance to benzimidazole configuration fungicides. Phytopathology 64:1139-1142.

25. Warren, C. G., Sanders, P., Cole, H. J., and Duich, J. M. 1977. Relative fitness of benzimidazole- and cadmium-tolerant populations of Sclerotinia homoeocarpa in the absence and presence of fungicides. Phytopathology 67:704-708.

26. Williams, D. W., Powell, A. J., Jr., and Vincelli, P. 1996. Dollar spot on bentgrass influenced by displacement of leaf surface moisture, nitrogen, and clipping removal. Crop Sci. 36:1304-1309.

27. Zietkiewicz, E., Rafalski, A., and Labuda, D. 1994. Genome fingerprinting by simple sequence repeat (SSR)-anchored polymerase chain reaction amplification. Genomics 20:176-183. 